\title{
Somatostatin Receptor 2 and 5 Expressions in Gastroenteropancreatic Neuroendocrine Tumors in Turkey
}

\author{
Omer Yerci ${ }^{1}$, Ibrahim Sehitoglu ${ }^{2 *}$, Nesrin Ugras $^{1}$, Erdem Cubukcu ${ }^{3}$, Suleyman \\ Yuce $^{4}$, Recep Bedir ${ }^{2}$, Erkan Cure ${ }^{4}$
}

\begin{abstract}
Background: Gastroenteropancreatic neuroendocrine tumors (GNs) are slow growing and although their incidence has increased in recent years, they are relatively rarely seen. Somatostatin analogues are used in the treatment of GNs that express somatostatin receptor (SR). We aimed to investigate the expression of SR2 and SR5 in GNs. Materials and Methods: In this study the expression of SR2 and SR5 was investigated immunohistochemically in 49 cases ( 26 males, 23 females) diagnosed and graded with GN according to the World Health Organization classification 2010. Results: The percentage of SR2 staining was $91.0 \%$ in grade 1,82.8\% in grade 2 and $100 \%$ in grade 3. On the other hand, the percentage of SR5 staining was $81.8 \% \%$ in grade 1 , $60.0 \%$ in grade 2 and $0 \%$ in grade 3 . According to the tumor localization, the percentages of SR2 expression were as follows: pancreas $85.7 \%$, stomach $100 \%$, small bowel $70 \%$, appendix $85.7 \%$ and rectum $100 \%$. The percentages of SR5 expression were: pancreas $61,9 \%$, stomach $37.5 \%$, small bowel 70\%, appendix $71.5 \%$ and rectum $66.6 \%$. There was a significant negative correlation between ki67 percentage and SR5 expression $(r=-0.341, p=0.016)$. Conclusions: In this study, GNs were found to highly express SR2 and SR5. Although the expression of SR2 and SR5 changed according to tumor localization, the expression of SR2 was higher than the expression of SR5 in GN. There was a significant negative correlation between ki67 and SR5. Accordingly, SR5 may be a prognostic indicator of GN.
\end{abstract}

Keywords: Gastroenteropancreatic neuroendocrine tumors - somatostatin receptor 2 - somatostatin receptor 5 - ki67

Asian Pac J Cancer Prev, 16 (10), 4377-4381

\section{Introduction}

Even though gastroenteropancreatic neuroendocrine tumors (GNs) are seen in various locations in the body, they are mostly found in gastrointestinal system (GIS). They are rare tumors originate from the diffuse neuroendocrine system cells and their incidences increase in recent years (Liu et al., 2013; Zeng et al., 2013). The presence of secretion function of tumor cells and due to the exhibition characteristics similar to the cells of neural origin they are called neuroendocrine tumors (Kloppel et al., 2005). Even though the secretion of biologically active peptides, amines and hormones cause the clinical symptoms of the patients (diarrhea, sweating, flushing), they may be inactive (Modlin et al., 2010). GNs are rare tumors that represent approximately $2 \%$ of GIS tumors and have an increased prevalence in recent years (Modlin et al., 2008). Due to the slow growth of GNs, they are difficult to be detected before being clinically symptomatic (Akerstrom et al., 2007; Kloppel et al., 2007).

Somatostatin, which inhibits tumor development by its antiangiogenic and anti-proliferative effect, is released widely from pancreas, GİS and central nervous system. It exerts its peripheral effects by 5 receptors (Guo et al., 2013). Due to the excessive expression of somatostatin receptors by GNs, somatostatin analogues (SAs) are used in their treatments (Oberg et al., 2004). SAs exert their effects including antineoplastic effects via somatostatin receptors (Koc et al., 2013). In literature, it has been reported that neuroendocrine tumors (NTs) express somatostatin receptors (SRs) in various rates and these receptors have been reported to prevent tumor development by induction of apoptosis in tumor cells (Papotti et al., 2002; Volante et al., 2007; Sclafani et al., 2011 ). Ki67 indicates active cells during cell cycle. It is an important cell marker that shows cell proliferation and is an important prognostic factor for various malignancies (Kilickap et al.,2014; Zhou et al., 2015). Additionally the percentage of ki67 proliferation is an important parameter in World Health Organization (WHO) 2010 classification of GN (Rindi et al., 2010).

In our country, there is only one study (Yeniay et al., 2012) that has been performed according to WHO 2000 classification, most cases had GNs localized in pancreas,

${ }^{1}$ Department of Pathology, ${ }^{3}$ Department of Medical Oncology, Faculty of Medicine, Uludag University, Bursa, ${ }^{2}$ Department of Pathology, ${ }^{4}$ Department of Internal Medicine, Faculty of Medicine, Recep Tayyip Erdogan University, Rize, Turkey *For correspondence: sehitogluibrahim@gmail.com 


\section{Omer Yerci et al}

and the expression of SR2 only was investigated. We aimed to have the country data by taking wider and variously localized GNs and investigating immunohistochemically the expression of SR2 and SR5. Also we purposed to investigate the relationship of clinicopathological data between SR2 and SR5 expression in GN

\section{Materials and Methods}

This study was compiled to the principles outlined in the Declaration of Helsinki and was affirmed by the ethics committee of Uludag University. This study was conducted in pathology department of Uludag University Medical Faculty between 1999-2010 and included 49 cases (26 males,23 females) diagnosed with GN. After taking all the preparations stained by Hematoxylineosin of all cases from the archives of the pathology department they were all reevaluated for diagnosis and histologic grade according to WHO 2010 classification. All the patients' information including gender, age, tumor localization and tumor diameter were obtained from the archived pathology reports.

\section{Histopathological method}

Sections of 4 micron were obtained from the patients paraffin blocks and were put in oven $\left(50-55^{\circ} \mathrm{C}\right)$ for one night then they were deparaffinized using xylene for 20 minutes. The sections were passed through alcohol series then washed by water for 5 minutes then were stored in distilled water for 10 minutes. The sections were then put into $10 \%$ citrate buffer, the sections were put in microwave oven and were boiled for 5 minutes in $800 \mathrm{~W}$ and 15 minutes in $400 \mathrm{~W}$. They were then allowed to cool for 20 minutes at room temperature and put into $3 \% \mathrm{H} 2 \mathrm{O} 2$ for 15 minutes. After being washed by distilled water they

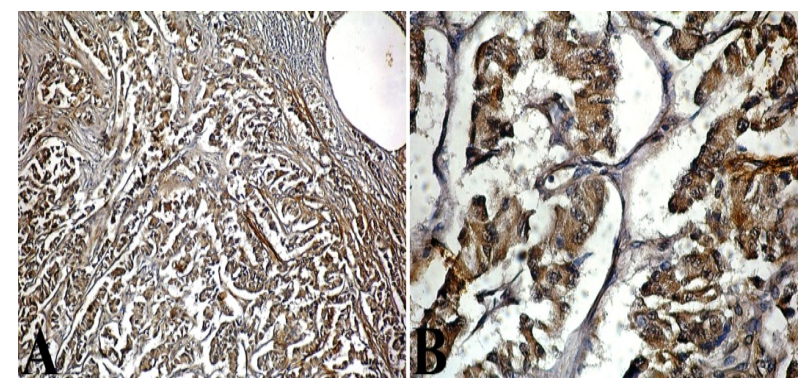

Figure 1. Immunoreactivity of Somatostatin Receptor 2 in Tumor. A: Diffuse Cytoplasmic Staining in Neoplastic Cells (x100) B: Strong Cytoplasmic Staining in Neoplastic Cells (x400)

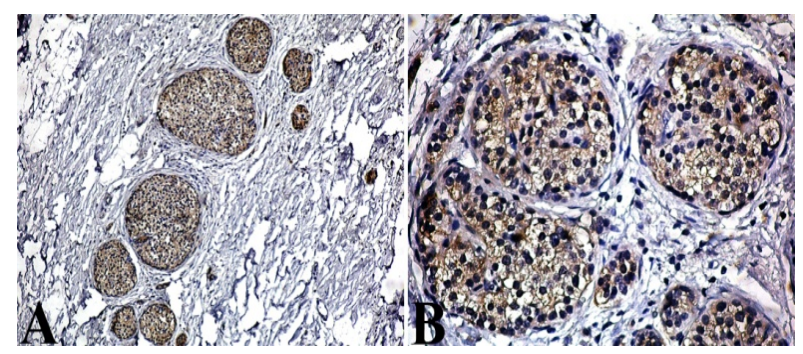

Figure 2. Immunoreactivity of Somatostatin Receptor 5 in Tumor. A: Diffuse Cytoplasmic Staining in Neoplastic Cells (x100) B: Strong Cytoplasmic Staining in Neoplastic Cells (x400) were put in phosphate buffered salin (PBS) for 10 minutes then they were allowed to stand for 10 minutes after a protein blockage was dropped. Every preparation was allowed to stand for 60 minutes after dropping primary antibodies (somatostatin receptor 2 and somatostatin receptor 5, ready-to-use mouse polyclonal antibodies, Novus Biologicals, Littleton, CO, USA). Then the sections were washed firstly by distilled water then by running water before being put into PBS for 10 minutes. Then they were incubated with Streptavidin-Horseradish Peroxidase (Universal LSAB Kit-K0690, DAKO). Antibody binding sites were stained with 3,3- diaminobenzidine and Mayer's hematoxylin was used as a counterstain for 4-5 minutes. PBS was used a negative control. The treated sections were examined under light microscope (BX51; Olympus, Tokyo, Japan). Employing a blind evaluation protocol performed by two pathologists, the immunoreactivity of SR2 and SR 5 were evaluated by semi-quantitative scoring system. The extent of staining was accepted as positive (more than $25 \%$ of stained cells), focally positive (less than $25 \%$ of stained cells, heterogeneously distributed), and negative (absence of staining) (Zamora et al., 2010)

\section{Statistical analysis}

Numerical data such as age and tumor size were expressed as mean $\pm \mathrm{SD}$. The other data were given as percentage and $\mathrm{n}$. Statistical tests were performed using SPSS (version 18, USA). Data that was expressed as categorical or $n$ were analyzed by chi square test. Mann Whitney U test was used for the statistical comparison of the other data. Pearson test was used for the correlation analysis. P level $<0.05$ was considered to be significant.

Table 1. Somatostatin Receptor 2 and 5 Expressions According to the Tumor Grades and Clinicopathological Parameters

\begin{tabular}{lccc}
\hline & $\begin{array}{c}\text { Grade 1 } \\
(\mathrm{n}=11)\end{array}$ & $\begin{array}{c}\text { Grade 2 } \\
(\mathrm{n}=35)\end{array}$ & $\begin{array}{c}\text { Grade 3 } \\
(\mathrm{n}=3)\end{array}$ \\
\hline Age (mean \pm SD) & $59.1 \pm 10.9$ & $47.1 \pm 19.4$ & $61.6 \pm 8.7$ \\
Gender (M/F) & $4-7$ & $16-19$ & $3-00$ \\
Tumor diameter cm & $1.2 \pm 0.8$ & $2.2 \pm 2.0$ & $4.0 \pm 3.4$ \\
Pancreas (n) & 3 & 17 & 1 \\
Stomach (n) & 2 & 5 & 1 \\
Small bowel (n) & 3 & 7 & 0 \\
Appendix (n) & 2 & 5 & 0 \\
Rectum (n) & 1 & 1 & 1 \\
SR2 expression (\%) & 91 & 82.8 & 100 \\
SR2 staining pattern & & & \\
$\quad$ None & 9 & 17.2 & 0 \\
Focally positive & 36.4 & 40 & 33.4 \\
$\quad$ Positive & 54.6 & 42.8 & 66.6 \\
SR5 expression(\%) & 81.8 & 60 & $0 ¥ \#$ \\
SR5 staining pattern & & & $*$ \\
$\quad$ None & 18.2 & 40 & 100 \\
Focally positive & 36.3 & 37.1 & 0 \\
Positive & 45.5 & 22.9 & 0 \\
Vascular invasion $(\%)$ & 9 & 28.5 & $66.6 \mathrm{~J}$ \\
Perineural invasion $(\%)$ & 18.1 & 17.1 & 33.3 \\
Lymph node metastasis $(\%)$ & 27.2 & 22.8 & $33.3 \neq$ \\
Necrosis (\%) & 9 & 8.5 & $66.6 \mathrm{~J} \chi$ \\
\hline
\end{tabular}

$* \mathrm{p}=0.025, ¥ \mathrm{p}=0.012$ vs grade $1 ; \ddagger \mathrm{p}=0.007, \chi \mathrm{p}=0.005, \# \mathrm{p}=0.048$, $\beta \mathrm{p}=0.025$ vs grade 2 


\section{Results}

A total of 49 cases ( 23 females and 26 males) were included in this study. Their mean age was $50.75 \pm 18.16$ years, the mean age of males was $51.69 \pm 16.35$ years and the mean age of females was $49.7 \pm 20.33$ years. There were 11 cases male/female $(\mathrm{M} / \mathrm{F})(7 / 4)$ with grade 1,35 cases male/female (M/F) (16/19) with grade 2, 3 cases male/female $(\mathrm{M} / \mathrm{F})(3 / 0)$ with grade 3 . The percentage of cases according to the grade was $22.4 \%$ for grade 1 , $71.4 \%$ for grade $2,6.2 \%$ for grade 3 . The percentage of SR2 staining was $91.0 \%$ for grade $1,82.8 \%$ for grade 2 and $100 \%$ for grade 3 (Figure 1). The percentage of SR5 staining was $81.8 \%$ for grade $1,60.0 \%$ for grade 2 and $0 \%$ for grade 3 (Figure 2). The percentages of SR2 and SR5 staining according to the grades and the data of other parameters according to the grades are given in table 1 .

The ratios of tumor localization of the cases were as follows; pancreas $42.8 \%$, stomach $16.3 \%$, small bowel
$20.4 \%$, appendix $14.3 \%$ and rectum $6.2 \%$. According to the tumor localization, the percentages of SR2 expression were as follows; pancreas $85.7 \%$, stomach $100 \%$, small bowel $70 \%$, appendix $85.7 \%$ and rectum $100 \%$. Whereas the percentages of SR5 expression were as follows; pancreas $61,9 \%$, stomach $37.5 \%$, small bowel $70 \%$, appendix $71.5 \%$ and rectum $66.6 \%$. The ratios of SR2 and SR5 expression according to the localization and other data were given in table 2 .

SR2 had no correlation with grade, ki67 percentage, tumor diameter, necrosis, lymphovascular invasion and perineural invasion. SR5 had no correlation with tumor diameter, necrosis, lymphovascular invasion and perineural invasion. However, there was a negative correlation between the expression of SR5 and ki67 percentage. Tumor diameter had a positive relation with grade, necrosis, vascular invasion and perineural invasion. The results of correlation analysis are shown in table 3 .

Table 2. Somatostatin Receptor 2 and 5 Expressions According to the Tumor Localization and Other Data

\begin{tabular}{|c|c|c|c|c|c|}
\hline & Pancreas $(n=21)$ & Stomach $(n=8)$ & Small bowel $(n=10)$ & Appendix $(n=7)$ & Rectum $(n=3)$ \\
\hline Age $($ mean \pm SD) & $48.5 \pm 16.4$ & $61.7 \pm 13.3$ & $58.8 \pm 14.6$ & $33.8 \pm 24.2 \#$ & $49.6 \pm 2.5$ \\
\hline Gender(M/F) (n) & $9-12$ & $3-5$ & $3-4$ & $4-5$ & $1-2$ \\
\hline Tumor diameter $\mathrm{cm}($ mean $\pm \mathrm{SD})$ & $2.2 \pm 1.6$ & $3.1 \pm 3.6$ & $2.2 \pm 1.2$ & $0.9 \pm 0.29 ¥$ & $0.8 \pm 0.5$ \\
\hline Grade $1(\%)$ & 14.3 & 25 & 30 & 28.5 & 33.4 \\
\hline Grade $2(\%)$ & 81 & 62.5 & 70 & 71.5 & 33.3 \\
\hline Grade $3(\%)$ & 4.7 & 12.5 & 0 & 0 & 33.3 \\
\hline SR2 expression (\%) & 85.7 & 100 & 70 & 85.7 & 100 \\
\hline SR2 staining pattern & & & & & $\beta$ \\
\hline None & 14.3 & 0 & 30 & 14.3 & 0 \\
\hline Focally positive & 52.4 & 62.5 & 20 & 28.6 & 0 \\
\hline Positive & 33.3 & 37.5 & 50 & 57.1 & 30 \\
\hline SR5 expression (\%) & 61.9 & 37.5 & 70 & 71.5 & 66.6 \\
\hline \multicolumn{6}{|l|}{ SR5 staining pattern } \\
\hline None & 38 & 62.5 & 30 & 28.5 & 33.4 \\
\hline Focally positive & 38 & 25 & 40 & 43 & 0 \\
\hline Positive & 24 & 12.5 & 30 & 28.5 & 66.6 \\
\hline Vascular invasion (\%) & 33.3 & 25 & 20 & 28.5 & 0 \\
\hline Perineural invasion (\%) & 19 & 25 & 30 & 0 & 0 \\
\hline Lymph node metastasis (\%) & 4.7 & 25 & 90 & $0 \mathrm{~W}$ & 0 \\
\hline Necrosis $(\%)$ & 9.5 & 25 & 20 & 0 & 0 \\
\hline
\end{tabular}

Table 3. Correlation Analysis Somatostatin Receptor 2 and 5 Expressions between Clinicopathological Parameters

\begin{tabular}{|c|c|c|c|c|c|c|c|c|c|c|c|c|c|c|}
\hline \multirow[b]{2}{*}{ Variable } & \multicolumn{2}{|c|}{ SR2 } & \multicolumn{2}{|c|}{ SR5 } & \multicolumn{2}{|c|}{ Ki-67 } & \multicolumn{2}{|c|}{$\begin{array}{c}\text { Tumor } \\
\text { diameter }\end{array}$} & \multicolumn{2}{|c|}{$\begin{array}{l}\text { Vascular } \\
\text { invasion }\end{array}$} & \multicolumn{2}{|c|}{$\begin{array}{l}\text { Lymph node } \\
\text { metastasis }\end{array}$} & \multicolumn{2}{|c|}{$\begin{array}{c}\text { Perineural } \\
\text { invasion }\end{array}$} \\
\hline & r value & $\mathrm{p}$ value & r value & $\mathrm{p}$ value & r value & $\mathrm{p}$ value & r value & $\mathrm{p}$ value & r value & $\mathrm{p}$ value & $\mathrm{r}$ value & $\mathrm{p}$ value & r value & $\mathrm{p}$ value \\
\hline Age & 0.082 & 0.575 & 0.069 & 0.637 & 0.145 & 0.319 & 0.180 & 0.216 & 0.033 & 0.822 & 0.272 & 0.059 & 0.266 & 0.066 \\
\hline Gender & 0.033 & 0.820 & 0.077 & 0.599 & 0.231 & 0.111 & 0.198 & 0.172 & 0.268 & 0.062 & 0.278 & 0.053 & 0.188 & 0.197 \\
\hline Tumor diameter & -0.031 & 0.831 & -0.040 & 0.785 & 0.334 & 0.019 & & & 0.402 & 0.004 & 0.236 & 0.102 & 0.560 & 0.001 \\
\hline $\mathrm{SR} 2$ & & & 0.085 & 0.589 & 0.100 & 0.493 & -0.031 & 0.831 & 0.245 & 0.089 & 0.154 & 0.290 & 0.043 & 0.769 \\
\hline SR5 & 0.085 & 0.589 & & & -0.341 & 0.016 & -0.040 & 0.785 & -0186 & 0.201 & 0.047 & 0.746 & 0.161 & 0.269 \\
\hline $\mathrm{Ki}-67$ & 0.100 & 0.493 & -0.341 & 0.016 & & & 0.334 & 0.019 & 0.316 & 0.027 & 0.350 & 0.014 & 0.144 & 0.323 \\
\hline Pancreas & 0.001 & 1.000 & 0.012 & 0.934 & 0.005 & 0.975 & 0.048 & 0.746 & 0.133 & 0.361 & 0.055 & 0.710 & 0.015 & 0.917 \\
\hline Stomach & 0.180 & 0.285 & 0.215 & 0.138 & 0.151 & 0.302 & 0.234 & 0.105 & 0.015 & 0.917 & 0.042 & 0.776 & 0.076 & 0.605 \\
\hline Small bowel & 0.227 & 0.116 & 0.091 & 0.533 & 0.108 & 0.461 & 0.032 & 0.828 & 0.075 & 0.609 & 0.220 & 0.129 & 0.152 & 0.297 \\
\hline Appendix & 0.001 & 1.000 & 0.084 & 0.559 & 0.125 & 0.391 & 0.241 & 0.095 & 0.019 & 0.989 & 0.154 & 0.290 & 0.194 & 0.182 \\
\hline Rectum & 0.104 & 0.476 & 0.029 & 0.846 & 0.141 & 0.332 & 0.161 & 0.270 & 0.153 & 0.292 & 0.032 & 0.826 & 0.121 & 0.407 \\
\hline Vascular invasion & 0.245 & 0.089 & -0186 & 0.201 & 0.316 & 0.027 & 0.402 & 0.004 & & & 0.035 & 0.812 & 0.312 & 0.029 \\
\hline Perineural invasion & 0.043 & 0.769 & 0.161 & 0.269 & 0.144 & 0.323 & 0.560 & 0.001 & 0.312 & 0.029 & 0.129 & 0.375 & & \\
\hline Lymph node metastasis & 0.154 & 0.290 & 0.047 & 0.746 & 0.350 & 0.014 & -0.236 & 0.102 & 0.035 & 0.812 & & & 0.129 & 0.375 \\
\hline Necrosis & 0.152 & 0.296 & 0.086 & 0.557 & 0.491 & 0.001 & 0.682 & 0.001 & 0.481 & 0.001 & 0.376 & 0.008 & 0.466 & 0.001 \\
\hline Grading & 0.018 & 0.902 & 0.017 & 0.909 & & & 0.425 & 0.002 & 0.203 & 0.163 & 0.552 & 0.001 & 0.049 & 0.740 \\
\hline
\end{tabular}




\section{Discussion}

It is known that the expression of SR in GN had antiproliferative, antimitotic and immunomodulator effects. In cases that express SR, SAs are used due to its antiproliferative and its inhibitor effects of hormonal release (Oberg K, 2001; Delaunoit et al., 2005; Demirkan et al., 2012). It has been reported that SAs show antiproliferative effect and suppressor effect of the tumor mediated hormone secretion in the presence of SR2 expression in acromegaly and carcinoid tumor, and in insulinoma with positive SR5 expression (Lamberts et al., 2002; Zhou et al 2014).

Few studies have reported GN to have various expression rates of SR2 and SR5 (Papotti et al., 2002; Volante et al., 2007; Sclafani et al., 2011; Zamora et al., 2009). Zhao et al. have reported $87.6 \%$ SR2 and $47.2 \%$ SR5 expressions in GN (Zhao et al., 2014). Volante et al. have reported 79\% SR2A expression in well differentiated neuroendocrine tumors/carcinomas, 44\% SR2A in poorly differentiated neuroendocrine carcinomas, $71 \%$ SR5 in well differentiated neuroendocrine tumors/carcinomas and $28 \%$ in poorly differentiated neuroendocrine carcinomas (Volante et al., 2007). Bertherat et al. have reported the expression of SR2 and SR 5 in cases with insulinoma to be $70 \%$ (Bertherat et al., 2003). In a study conducted by Iida et al. (2010) on patients with GN they have reported the expression of SR2A to be $90.6 \%$ and SR 5 to be $78.1 \%$ (Iida et al., 2010), Pisarek et al. (2010) have reported SR2A to be $38.9 \%$ and SR5 to be $58.8 \%$ (Pisarek et al., 2010) and Nakayama et al. (2010) have reported the expression of SR2 to be $81 \%$ in low-grade pancreas localized GN (Nakayama et al., 2010). In our study, we have found the expression of SR2 in GN to be respectively; pancreas $85.7 \%$, stomach $100 \%$, small bowel $70 \%$, appendix $85.7 \%$ and rectum $100 \%$. Whereas the expression of SR5 was pancreas $61.9 \%$, stomach $37.5 \%$, small bowel $70 \%$, appendix $71.5 \%$ and rectum $66.6 \%$. When investigating in details with the exception of Pisarek et al. study all the previous studies including our study we have found that SR5 positivity to be low in GN and the expression of SR5 to be decreasing with increasing the tumor grade (Pisarek et al., 2010).

In addition, in Zamora et al. study that was conducted on 100 cases with GN; 67 cases had gastrointestinal, 25 pancreatic and 8 liver metastasis the positivity of SR2 was $86 \%$ and SR5 was $62 \%$ (Zamora et al., 2009). The results of the study were very similar to our results. The most cases of their study had small bowel and pancreatic tumor localization. The authors have reported that SR2 and SR5 to be mostly positive in cases with nonpancreatic localized tumors and nonfunctional tumors and the positivity to be decreased with increasing the grade. Additionally the authors have reported the expression of SR5 to be decreased with increasing tumor grade similar to the results of most studies published in the literature (Zamora et al., 2009). On the contrary to the other studies in a study (de Sa et al., 2006) conducted on insulinoma the positivity of SR4 was 22\%, SR2 was $89 \%$ and SR1-3-5 was $100 \%$. The authors have reported SR5 to be correlated with tumor diameter and tumor aggressiveness (de Sa et al., 2006). That was an interesting information. In fact, SR5 is well known to inhibit cell proliferation and insulin release. The cases selection and their number might affect the results of their study. Because their result regarding SR5 expression was not compatible with the literature (Volante et al., 2007; Zamora et al., 2009) .

Interestingly, Zhao et al. in their wide series study have reported SR2 to be a negative prognostic indicator for GN (Zhao et al., 2014). However, 90\% of the cases had stomach and small bowel localized NT and SR5 expression was very low (\%47.2). The results of our study are similar to Zhao et al. study that SR5 expression was very low as $37.5 \%$ in GN. However, in our study GNs were $42.8 \%$ pancreas localized and in various studies it has been shown that SR2 and SR5 to be expressed in high rate in pancreas localized NT (Papotti et al., 2002; Bertherat et al., 2003). Additionally while there was no colon localized NT in our study, there was $4.6 \%$ cases with colon localization in Zhao et al. study (Zhao et al., 2014). Moreover; their cases with pancreas-localized tumor was very low. So they might have found low SR5 positivity and due to the lack of similar cases profile they might found SR2 to be prognostic for GN. In our study, we have not found correlation between SR2 and ki67. That may be associated with the localization and the number of cases. While SR2 had a similar staining pattern in grade1, 2 and 3, SR5 had lower staining percentage with increasing tumor grade. We have found a negative correlation between SR5 expression and ki67 percentage. Whereas high ki67 proliferation index is an important prognostic factor in many tumors (Kilickap et al.,2014; Zhou et al., 2015) , it has a special importance in GN. The reason is that the percentage of ki67 proliferation index is used in grading and the determination whether to give chemotherapy in GN (Rindi et al., 2010). Beside the importance of SR2 and SR5 in the treatment of GN and in the contrary to Zhao et al. study (Zhao et al., 2014) SR5 positivity may be an important prognostic factor especially in NT localized to pancreas.

Yeniay et al. have investigated the prognostic importance of SR2 expression in GN (Yeniay et al., 2012). In their study, SR2 was found to have no correlation with lymph node metastasis, necrosis, vascular invasion or perineural invasion. To our knowledge their study is the first to be conducted on GN in our country and only the expression of SR2 was investigated; thus our study is considered to be the first study in our country that investigate both SR2 and SR5 expression. In our study, we have not found both SR2 and SR5 expressions to be related with lymph node metastasis, necrosis, vascular invasion, or perineural invasion. However, tumor grade had positive correlation with lymph node metastasis and tumor diameter. We have found that tumor diameter increase with increased rate of vascular and perineural invasion. In literature, the incidence of nonfunctional GNs has been reported to be increasing (Halfdanarson et al., 2008). Due to their clinically asymptomatic course, nonfunctional tumors are detected mostly when being metastasized. In the current study, the status of hormonal secretion of the cases was not investigated. In fact large diameter of tumors that have vascular invasion and 
perineural invasion may suggest them to nonfunctional.

While the expression of SR2 is highly observed, SR5 expression is seen less in GN. The expression rate of SR2 and SR5 changes according to tumor localization. SAs show anti-proliferative effect and an inhibitory effect of the hormone secretion in these tumors. Therefore, SR2 and SR5 expressions are important for the treatment of GNs. In conclusion, due to the negative correlation that we have found between SR5 expression and ki67 percentage, SR5 may be a prognostic indicator in these tumors.

\section{References}

Akerström G, Hellman P (2007). Surgery on neuroendocrine tumours. Best Pract Res Clin Endocrinol Metab, 21, 87-109.

Bertherat J, Tenenbaum F, Perlemoine K, et al (2003). Somatostatin receptors 2 and 5 are the major somatostatin receptors in insulinomas: an in vivo and in vitro study. JClin Endocrinol Metab, 88, 5353-60.

Delaunoit T, Rubin J, Neczyporenko F, Erlichman C, Hobday TJ (2005). Somatostatin analogues in the treatment of gastroenteropancreatic neuroendocrine tumors. Mayo Clin Proc, 80, 502-6.

Demirkan BH, Eriksson B (2012). Systemic treatment of neuroendocrine tumors with hepatic metastases. Turk $J$ Gastroenterol, 23, 427-37.

de Sá SV, Corrêa-Giannella ML, Machado MC, et al (2006). Somatostatin receptor subtype 5 (SSTR5) mRNA expression is related to histopathological features of cell proliferation in insulinomas. Endocr Relat Cancer, 13, 69-78.

Guo RS, Shi PD, Zhou J, Chen YY (2013). Somatostatin receptors 3, 4 and 5 play important roles in gallbladder cancer. Asian Pac J Cancer Prev, 14, 4071-5.

Halfdanarson TR, Rubin J, Farnell MB, Grant CS, Petersen GM (2008). Pancreatic endocrine neoplasms: Epidemiology and prognosis of pancreatic endocrine tumors. Endocr Relat Cancer, 15, 409-27.

Iida S, Miki Y, Ono K, et al (2010). Novel classification based on immunohistochemistry combined with hierarchical clustering analysis in non-functioning neuroendocrine tumor patients. Cancer Sci, 101, 2278-85.

Kilickap S, Kaya Y, Yucel B, Tuncer E, Babacan NA, Elagoz S (2014). Higher Ki67 expression is associates with unfavorable prognostic factors and shorter survival in breast cancer. Asian Pac J Cancer Prev, 15, 1381-5.

Kloppel G, Anlauf M (2005). Epidemiology, tumour biology and histopathological classification of neuroendocrine tumours of the gastrointestinal tract. Best Pract Res Clin Gastroenterol, 19, 507-17.

Kloppel G, Rindi G, Anlauf M, Perren A, Komminoth P (2007). Site-specific biology and pathology of gastroenteropancreatic neuroendocrine tumors. Virchows Arch, 451, 9-27.

Koc EU, Ozgur T, Yerci O, Gurel S (2013). Somatostatin receptor 1 (SSTR1) and somatostatin receptor 5 (SSTR5) expression in hepatocellular carcinoma. Hepatogastroenterology, $6 \mathbf{0}$, 1693-7.

Lamberts SW, de Herder WW, Hofland LJ (2002). Somatostatin analogs in the diagnosis and treatment of cancer. Trends Endocrinol Metab, 13, 451-7.

Liu SZ, Zhang F, Chang YX, et al (2013). Prognostic impact of cyclin D1, cyclin E and P53 on gastroenteropancreatic neuroendocrine tumours. Asian Pac J Cancer Prev, 14, 419-22.

Modlin IM, Oberg K, Chung DC, et al (2008). Gastroenteropancreatic neuroendocrine tumours. Lancet Oncol, 9, 61-72.
Modlin IM, Moss SF, Oberg K, et al (2010). Gastrointestinal neuroendocrine (carcinoid) tumours: current diagnosis and management. Med J Aust, 193, 46-52.

Nakayama Y, Wada R, Yajima N, Hakamada K, Yagihashi S (2010). Profiling of somatostatin receptor subtype expression by quantitative PCR and correlation with clinicopathological features in pancreatic endocrine tumors. Pancreas, 39, 1147-54.

Oberg K (2001). Chemotherapy and biotherapy in the treatment of neuroendocrine tumours. Ann Oncol, 12, 111-4.

Oberg K, Kvols L, Caplin M, et al (2004). Consensus report on the use of somatostatin analogs for the management of neuroendocrine tumors of the gastroenteropancreatic system. Ann Oncol, 15, 966-73.

Papotti M, Bongiovanni M, Volante M, et al (2002). Expression of somatostatin receptor types 1-5 in 81 cases of gastrointestinal and pancreatic endocrine tumors. A correlative immunohistochemical and reverse-transcriptase polymerase chain reaction analysis. Virchows Arch, 440, 461-75.

Pisarek H, Pawlikowski M, Kunert-Radek J, Kubiak R, Winczyk K (2010). SSTR1 and SSTR5 subtypes are the dominant forms of somatostatin receptor in neuroendocrine tumors. Folia Histochem Cytobiol, 48, 142-7.

Rindi G, Arnold R, Bosman FT, et al (2010). Nomenclature and classification of neuroendocrine neoplasms of the digestive system, Eds Bosman FT, Carnerio F, Hruban RH, Theise ND, WHO classification of tumors of the digestive system. Fourth ed. WHO press, Lyon, pp. 13-4 .

Sclafani F, Carnaghi C, Di Tommaso L, et al (2011). Detection of somatostatin receptor subtypes 2 and 5 by somatostatin receptor scintigraphy and immunohistochemistry: clinical implications in the diagnostic and therapeutic management of gastroenteropancreatic neuroendocrine tumors. Tumori, 97, 620-8.

Volante M, Brizzi MP, Faggiano A, et al (2007). Somatostatin receptor type $2 \mathrm{~A}$ immunohistochemistry in neuroendocrine tumors: a proposal of scoring system correlated with somatostatin receptor scintigraphy. Mod Pathol, 20, 1172-82.

Yeniay L, Gurcu B, Unalp O, et al (2012). Prognostic value of somatostatin receptor-2 positivitiy in gastroenteropancreatic neuroendocrine tumors in reference to known prognostic factors. Turk J Gastroenterol, 23, 736-40.

Zamora V, Cabanne A, Salanova R, et al (2010). Immunohistochemical expression of somatostatin receptors in digestive endocrine tumours. Dig Liver Dis, 42, 220-5.

Zeng YJ, Liu L, Wu H, et al (2013). Clinicopathological features and prognosis of gastroenteropancreatic neuroendocrine tumors: analysis from a single-institution. Asian Pac J Cancer Prev, 14, 5775-81.

Zhao WY, Zhuang C, Xu J, et al (2014). Somatostatin receptors in gastrointestinal stromal tumors: new prognostic biomarker and potential therapeutic strategy. Am J Transl Res, 6, 831-40.

Zhou G, Sinnett-Smith J, Liu SH, et al (2014). Front Physiol, $5,226$.

Zhou Y, Jiang HG, Lu N, Lu BH, Chen ZH (2015). Expression of ki67 in papillary thyroid microcarcinoma and its clinical significance. Asian Pac J Cancer Prev, 16, 1605-8. 